\title{
Modeling a turbulence effect in formation of the Antarctic Circumpolar Current
}

\author{
J. Heinloo and A. Toompuu \\ Marine Systems Institute, Tallinn University of Technology, Akadeemia tee 21, Tallinn 12618, Estonia \\ Received: 3 March 2006 - Revised: 27 October 2006 - Accepted: 24 November 2006 - Published: 21 December 2006
}

\begin{abstract}
A simple model to determine a turbulence effect in formation of the Antarctic Circumpolar Current (ACC) is suggested. The model is founded on the theory of rotationally anisotropic turbulence and is set up as a generalization of the geostrophic description of the Antarctic Circumpolar Current (ACC). It predicts the turbulence effect as an additive correction to the flow velocity predicted by the geostrophic balance. The correction, calculated from the optimally analyzed hydrographic data in the Southern Ocean, results in an increased ACC total baroclinic volume transport and in a shift in the current velocity maximum to the south, if compared with the pure geostrophic estimate.
\end{abstract}

Keywords. Oeanography: physical (currents, general circulation; turbulence, diffusion and mixing processes)

\section{Introduction}

Since the classical work of Munk and Palmén (1951) the ACC is commonly tied to westerlies dominating in the region. The approach stems from the momentum balance with the momentum transfer by shear from the surface to the bottom, complemented by the meridional overturning (Johnson and Bryden, 1989; Morrow et al., 1992; Gille, 1997; Tansley and Marshall, 2001, etc.). An alternative approach by Wyrtki (1960), Baker (1982), Hellerman and Rosenstein (1983) and Mestaz-Nunez et al. (1992) is based on the Sverdrup balance. Both approaches are still intensively debated (Warren et al., 1996; Olbers, 1998; Gnanadesikan and Hallberg, 2000; Rintoul et al., 2001; Karsten et al., 2002).

The skill of the quoted approaches (wind-driven models of the ACC) appears to be limited when describing the ACC branched structure, with branches extending to significant depths and associated with the spatial structure of the frontal

Correspondence to: J. Heinloo

(heinloo@phys.sea.ee) regions in the Southern Ocean (Whitworth et al., 1982; Hofmann, 1985; Nowlin and Klinck, 1986; Phillips and Rintoul, 2000; Niiler, 2001). The limitation challenges other ACC models proceeding from the thermohaline structure of the region, where the southward density increase in the density frontal zones is accompanied by the ocean surface sloping up to the north. The latter, together with the varying density field, causes the pressure gradient directed to the north in the ocean interior down to an isopotential reference level. The geostrophic balance condition determines the eastward flow above the reference level with the velocity maxima in the density frontal zones. For the density field with several frontal zones the predicted velocity has a branched structure. Nevertheless, the pure geostrophic model of the ACC underestimates the actual ACC net volume transport. The latter is estimated from direct velocity measurements in the Drake Passage and in the southwestern Argentine Basin, above $3000 \mathrm{~m}$ depth, by different authors to be about $140 \mathrm{~Sv}$ while the geostrophic transport predicted by the geostrophic balance relative to $3000 \mathrm{~m}$ is only about $100 \mathrm{~Sv}$. A summary of the net transport estimates based on the direct velocity measurements and on the geostrophic balance relative to $3000 \mathrm{~m}$ depth can be found in Whitworth et al. (1982); Arhan et al. (2002). The difference is commonly explained by an eastward barotropic flow velocity constituent not predicted by the geostrophic balance (Whitworth et al., 1982). This paper suggests a turbulence-generated baroclinic mechanism contributing to the difference in the transport estimates.

The first attempt to discuss the turbulence effect in the ACC formation was made by Heinloo and Toompuu (2004). The discussion was based on the theory of rotationally anisotropic turbulence (RAT theory) (Heinloo, 2004), accounting for the preferred orientation of eddy rotation. Although the model that was suggested in Heinloo and Toompuu (2004) resulted in a turbulence-generated eastward zonal flow associated with density fronts, it disregarded the geostrophic constituent of the flow velocity. Contrary to the

Published by Copernicus GmbH on behalf of the European Geosciences Union. 
cited paper the current paper treats the turbulence effect as an ageostrophic correction to the velocity predicted by the geostrophic balance. In order to outline the turbulence influence more explicitly, the model disregards the effects of momentum, heat and fresh water fluxes across the upper boundary (ocean surface), as well as the barotropic constituent of the flow velocity.

The model predictions are illustrated by the turbulencecorrected volume transport of the ACC and by the meridional distribution of the depth-integrated flow velocity calculated from the gridded hydrographic data in the Southern Ocean (Olbers et al., 1992). The calculations show that the turbulence-corrected volume transport of the ACC exceeds the transport predicted from the pure geostrophic balance. The increase of the volume transport of the ACC is accompanied with a shift in the velocity maximum(s) in the meridional sections to the south.

\section{The RAT theory}

The RAT theory (Heinloo, 2004) modifies the conventional turbulence mechanics (CTM) by accounting for the preferred orientation of eddy rotation. The modification is formalized by including the curvature radius $\boldsymbol{R}$ of the streamline of the turbulent flow velocity $\boldsymbol{v}$ and the fluctuating constituent $\boldsymbol{v}^{\prime}$, into the arguments of the probability distribution which specifies the applied statistical averaging. The modification enables the introduction of the moment of momentum or angular momentum of the turbulent flow field

$\boldsymbol{M}=\left\langle\boldsymbol{v}^{\prime} \times \boldsymbol{R}\right\rangle$,

reflecting the average effect of the eddies' rotation preferred orientation without any explicit determination of the eddies. The definition of $\boldsymbol{M}$, determined at each flow field point, is coupled with the definition

$\boldsymbol{\Omega}=\left\langle\boldsymbol{v}^{\prime} \times \frac{\boldsymbol{R}}{R^{2}}\right\rangle$.

Quantity $\boldsymbol{\Omega}$ is independent from the average velocity $\boldsymbol{u}=\langle\boldsymbol{v}\rangle$ kinematical characteristic of the flow field with the dimension of the angular velocity of rotation. For the turbulent media, where $\boldsymbol{M} \neq 0$ (or $\boldsymbol{\Omega} \neq 0$ ) (the turbulent media with $\boldsymbol{M} \neq 0$ are called "rotationally anisotropic"), the turbulence energy $K^{=} \frac{1}{2}\left\langle v^{\prime 2}\right\rangle$ is divided into the sum $K=K^{\Omega}+K^{0}$, where $K^{\Omega}=\frac{1}{2} \boldsymbol{M} \cdot \boldsymbol{\Omega}$ and $K^{0}=\frac{1}{2}\left\langle\boldsymbol{M}^{\prime} \cdot \boldsymbol{\Omega}^{\prime}\right\rangle$ in which $\boldsymbol{M}^{\prime}=\boldsymbol{v}^{\prime} \times$ $\boldsymbol{R}-\boldsymbol{M}$ and $\boldsymbol{\Omega}^{\prime}=\boldsymbol{v}^{\prime} \times \boldsymbol{R} / R^{2}-\boldsymbol{\Omega}$.

The setup of the description of turbulent flows with $\boldsymbol{M} \neq 0$ is grounded on the conservation laws for the average momentum (the Reynolds equation), for the moment of momentum $\boldsymbol{M}$ describing the orientated (large-scale) turbulence constituent and for energy $K^{0}$, describing the unorientated (small-scale) turbulence constituent. Due to the interaction between the average flow and the orientated (large-scale) turbulence constituent, the turbulent (Reynolds) stress tensor
$R_{i j}=-\rho\left\langle v_{i}^{\prime} v_{j}^{\prime}\right\rangle$ (here and henceforth the Latin indices have the values $1,2,3$ ) becomes asymmetric, with the antisymmetric constituent describing the interaction. The asymmetry of the turbulent stress tensor means that for $\boldsymbol{M} \neq 0$ the probability distribution applied becomes non-invariant with respect to the commutation of its arguments, $v_{i}^{\prime}$ and $v_{j}^{\prime}$. The antisymmetric constituent of the turbulent stress tensor vanishes, together with $\boldsymbol{M}, \boldsymbol{\Omega}$ and $K^{\Omega}$, if either the averaging is treated in the way adopted within the CTM or $\boldsymbol{M}, \boldsymbol{\Omega}$ and $K^{\Omega}$ all vanish due to the absence of the preferred orientation of the eddy rotation. In both cases, the RAT theory reduces to the CTM. The RAT theory, as a generalization of the CTM, assigns the turbulent media with additional properties not reflected by the CTM. One of the additional properties, playing an essential role in the ACC model discussed below, is the rotational viscosity, which is revealed in the relative rotation, i.e. when $\boldsymbol{\Omega} \neq \boldsymbol{\omega}$, where $\boldsymbol{\omega}=\frac{1}{2} \nabla \times \boldsymbol{u}$ is the vorticity, and is characterized by the coefficient of the rotational viscosity $\gamma$, which connects the dual vector $\sigma$ of the antisymmetric constituent of the Reynolds stress tensor, determined by its components $\sigma_{k}=\frac{1}{2} e_{i j k} R_{i j}$, where $e_{i j k}$ are the components of the Levi-Civita tensor, with the angular velocity of the relative rotation,

$\sigma=4 \gamma(\boldsymbol{\Omega}-\boldsymbol{\omega})$.

\section{The model}

\subsection{The model background}

The model determines the ACC as a flow in the Southern Ocean, encircling the Antarctic continent in the clockwise direction, as viewed from the South Pole. The flow is assumed to be stationary with the velocity field $\boldsymbol{u}=(u(\vartheta, z), 0,0)$ in spherical coordinates $(\varphi, \vartheta, z)$ where $\varphi$ is longitude, $\vartheta$ is the south latitude and depth $z$ is positive downward. The potential density $\rho=\rho(\vartheta, z)$ (henceforth - the density) is determined in the flow region as $\rho=\rho_{0}+\rho^{*}$, in which $\rho_{0}=10^{3} \mathrm{~kg} \mathrm{~m}^{-3}$ and $\rho^{*}<<\rho_{0}$. The assumptions of the Boussinesq approximation (Pedlosky, 1982) are adopted.

\subsection{Geostrophic or "thermal wind" model of the ACC}

The geostrophic or "thermal wind" model of the ACC stems from the balance condition

$-\nabla p+2 \rho_{0} \boldsymbol{u} \times \omega^{0}+\rho \boldsymbol{g}=0$,

( $p$ denotes pressure, $\omega^{0}=\left(0,-\omega^{0} \cos \vartheta, \omega^{0} \sin \vartheta\right.$ ) is the angular velocity of the Earth's rotation and $\boldsymbol{g}=(0,0, g)$ is the gravitational acceleration), where, in accordance with the geostrophic approximation of oceanographic flows (Pedlosky, 1982), the vertical constituent of the Coriolis force is considered as negligibly small with respect to $\rho g$ and $p_{, z}$ (here and henceforth the index after the comma denotes the partial derivative along the corresponding coordinate). The 
assumption made allows one to replace the vertical projection of Eq. (4) with $p_{, z}=\rho g$, from which $p=p_{0}-g \int_{z}^{H} \rho d z$, where $H$ is the depth of the reference (isopotential) level and $p_{0}$ is the constant pressure at the depth $z=H$. Using $p=p_{0}-g \int_{z}^{H} \rho d z$ from the meridional projection of Eq. (4), we obtain

$u=C_{1} \frac{\partial}{\partial \vartheta}\left(\frac{1}{\rho_{0} H} \int_{z}^{H} \rho d z\right)$.

In Eq. (5) $C_{1}=g H / 2 r \omega^{0} \sin \vartheta$, where $r$ is the Earth's radius. As applied to the ACC region, Eq. (5) determines the eastward flow down to the isopotential reference level $z=H$.

\subsection{Ageostrophic model of the ACC}

\subsubsection{Ageostrophic velocity constituent of the flow field}

In the following the outlined geostrophic model of the ACC is generalized to account for the turbulence effects. The generalization is founded on the RAT theory considered within the Richardson-Kolmogoroff concept about the cascading structure of turbulent eddies (Richardson, 1922; Kolmogoroff, 1941). The concept allows one to disregard the effect of the shear viscosity (reflecting the immediate interaction between the average flow and the small-scale turbulence with energy $K^{0}$ ), if compared with the effect of the rotational viscosity (reflecting the interaction between the average flow and the large-scale turbulence with energy $K^{\Omega}$ ).

Representing the flow velocity as the sum $\boldsymbol{u}=\boldsymbol{u}_{g}+\boldsymbol{u}_{a g}$, where $\boldsymbol{u}_{g}=\left(u_{g}, 0,0\right)$ denotes the flow velocity constituent predicted by the geostrophic approach and $\boldsymbol{u}_{a g}=\left(u_{a g}, 0,0\right)$ is the flow velocity constituent arising due to the assumed turbulence effect, the momentum equation of the RAT theory (Heinloo, 2004), written for the motion in the frame rotating together with the Earth, reads as

$$
-\nabla p+\frac{1}{2} \nabla \times \boldsymbol{\sigma}+2 \rho_{0}\left(\boldsymbol{u}_{g}+\boldsymbol{u}_{a g}\right) \times \omega^{0}+\rho \boldsymbol{g}=0,
$$

in which the divergence of the turbulent (Reynolds) stress tensor is expressed as $\frac{1}{2} \nabla \times \sigma$. It is easy to conclude from Eq. (6) that only the longitudinal component of $\sigma$ can affect the flow, therefore it is assumed further that $\sigma=(\sigma, 0,0)$. Taking into account that the longitudinal component of $\omega$ is zero, from Eq. (3) we have for $\boldsymbol{\sigma}$

$\sigma=4 \gamma \boldsymbol{\Omega}_{\varphi}$,

where $\boldsymbol{\Omega}_{\varphi}=(\Omega, 0,0)$.

Consider first the case when condition $p_{, z}=\rho g$ holds exactly. When the turbulent effects are absent and the meridional component of the planetary vorticity cannot be neglected, then this condition determines $u_{g}$ to be vanishing.
Contrary to $u_{g}$, the ageostrophic constituent of the flow velocity $u_{a g}$ does not vanish in this case and is determined from the vertical projection of Eq. (6) as

$u_{a g}=-\frac{\gamma}{r \rho_{0} \omega^{0} \cos ^{2} \vartheta}(\Omega \cos \vartheta)_{, \vartheta}$,

coinciding with velocity $u$. Result Eq. (8) with $u_{a g}=u$ corresponds to the model discussed in Heinloo and Toompuu (2004). Unlike that in the discussed case, if the balance condition $p_{, z}=\rho g$ is considered to be holding to the same degree of accuracy as in the geostrophic approach, then $u_{g}$ does not vanish and velocity $u$ appears as the sum of geostrophic and ageostrophic constituents, $u=u_{g}+u_{a g}$. This modified treatment would reduce either to the model of Heinloo and Toompuu (2004), if $u_{g}=0$, or to the geostrophic or "thermal wind" model outlined in Sect. 3.2, if the turbulence effect is absent. For the rotational viscosity present, the latter situation would take place if $\Omega=0$. (For $u$ determined according to the discussion presented above, the meridional projection of Eq. (6) should be applied to determine the meridional pressure gradient.)

To determine $\Omega$ in Eq. (8) the zonal projection of the equation for the moment of momentum $\boldsymbol{M}$ is considered. Neglecting the term describing the diffusion of $\boldsymbol{M}$, the zonal projection of the equation for the moment of momentum $\boldsymbol{M}$ (Heinloo, 2004) reads as

$-\sigma-4 \kappa \Omega+\rho_{0} m=0$.

The first term in Eq. (9) is defined by Eq. (7) as $\sigma=4 \gamma \Omega$, the second term is the zonal projection of the moment associated with the decay of $\boldsymbol{M}$ due to the moment of momentum scatter in the cascading process $(\kappa>0$ is the coefficient of the cascade scattering) and the third term is the $\varphi$-component of the body moment $\rho_{0}\left\langle\boldsymbol{f}^{\prime} \times \boldsymbol{R}\right\rangle$ caused by the body force fluctuations $f^{\prime}$. For the model background stated in Sect. 3.1, $\boldsymbol{f}^{\prime}$ coincides with the reduced gravity $\boldsymbol{g}^{\prime}=\rho^{\prime} \boldsymbol{g} / \rho_{0}$, where $\rho^{\prime}$ denotes the density fluctuation. Using closure $\left\langle\rho^{\prime} \boldsymbol{R}\right\rangle=k_{1} \nabla \rho \times \boldsymbol{\Omega}-k_{2} \nabla \rho$, where $k_{1}$ and $k_{2}$ are constants, deduced as the dependence of $\left\langle\rho^{\prime} \boldsymbol{R}\right\rangle$ on $\boldsymbol{\Omega}$ and $\nabla \rho$ linear with respect to either of the arguments and equal to zero for $\nabla \rho=0$, we have for $\rho_{0} m$

$\rho_{0} m=-k_{1} g \rho_{, z} \Omega+k_{2} \frac{g}{r} \rho_{, \vartheta}$.

If accounting for Eq. (10), then the balance condition (Eq. 9) can be expressed as

$-\sigma-4 \kappa \Omega-k_{1} g \rho_{, z} \Omega+k_{2} \frac{g}{r} \rho_{, \vartheta}=0$.

The term in Eq. (11), including $k_{1}$, is the moment acting on the medium particles due to the stratification. Insofar as the stable stratification $\left(\rho_{, z}>0\right)$ causes $\Omega$ to decrease and the unstable stratification $\left(\rho_{, z}<0\right)$ causes $\Omega$ to increase, the coefficient $k_{1}$ is always positive. The term in Eq. (11), including $k_{2}$, describes the contribution of baroclinic effect to the 


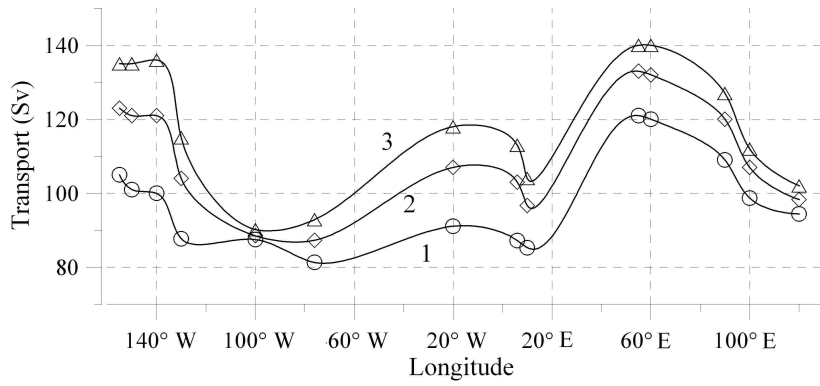

Fig. 1. The eastward baroclinic volume transport in the Southern Ocean between the southern boundary of the ACC and $30^{\circ} \mathrm{S}$ in the depth interval from 100 to $3000 \mathrm{~m}$ for pure geostrophic transport $Q_{g}$ with $A=0$ (curve 1) and for the corrected transport $Q$ with $b=10^{3}, A=6 \cdot 10^{-4} \mathrm{~m} \cdot \mathrm{s}^{-1}$ (curve 2), $b=10^{3}, A=10^{-3} \mathrm{~m} \cdot \mathrm{s}^{-1}$ (curve 3).

$\Omega$-field. The model considers the medium as baroclinically instable with the baroclinic instability being the only source of turbulence energy and therefore the model states $k_{2}$ to be also positive.

From Eq. (11) we have for $\Omega$,

$\Omega=\frac{k_{2} g}{r} \frac{\rho_{, \vartheta}}{4(\gamma+\kappa)+k_{1} g \rho_{, z}}$.

Finally, replacing $\Omega$ in Eq. (8) by Eq. (12) we have for $u_{a g}$,

$u_{a g}=-\alpha C_{2} \frac{1}{r^{2} \cos ^{2} \vartheta}\left(\frac{\rho_{, \vartheta}}{1+\beta g \rho_{, z}} \cos \vartheta\right)_{, \vartheta}$,

where $C_{2}=g / \rho_{0} \omega^{0}, \alpha=\gamma k_{2} / 4(\gamma+\kappa)$ and $\beta=k_{1} / 4(\gamma+\kappa)$. Expression (13) presents the ageostrophic velocity constituent as depending on two independent physical coefficient combinations, $\alpha$ and $\beta$. Ageostrophic velocity constituent $u_{a g}$ vanishes for $\gamma \neq 0$ only if $k_{2}=0$, i.e. if the medium is baroclinically stable. Then $u=u_{g}$ and the flow is governed by the geostrophic balance.

The argumentation presented above determines velocity $u$ with an accuracy up to an additive arbitrary barotropic (reference) velocity constituent, which cannot be specified within the suggested model. It should also be noted that the ageostrophic correction $u_{a g}$, Eq. (13), depends on up to the second derivative of the density distribution with respect to $\vartheta$ while the geostrophic constituent $u_{g}$, defined by Eq. (5), depends only on the first derivative.

\subsubsection{Calculations and discussion}

The potential density in the Southern Ocean area in the latitude band from $30^{\circ} \mathrm{S}$ to $80^{\circ} \mathrm{S}$ was calculated according to the international equation of state for seawater (Fofonoff and Millard, 1983) from long-term optimally analyzed and gridded data on temperature and salinity (Olbers et al., 1992) measured since the 1920s. The gridded data were available with the $1^{\circ} \times 1^{\circ}$ horizontal resolution for the depths $0,10,20$,
$30,40,50,75,100,125,150,200,250,300,350,400,500$, $600,700,800,900,1000,1100,1200,1300,1400,1500$, $1750,2000,2250,2500,2750,3000,3250,3500,3750$, 4000,4500 and $5000 \mathrm{~m}$. The geostrophic velocity $u_{g}$ was calculated according to Eq. (5) and the ageostrophic velocity constituent $u_{a g}$ according to

$u_{a g}=-\frac{A}{\cos ^{2} \vartheta}\left(\frac{\left(\rho / \rho_{0}\right)_{, \vartheta}}{1+b H\left(\rho / \rho_{0}\right)_{, z}} \cos \vartheta\right)_{, \vartheta}$,

which coincides with Eq. (13) for $A=C_{2} \rho_{0} \alpha / r^{2}$ and nondimensional $b=\rho_{0} g \beta / H$, where $H$ is identified with the reference depth. Calculations were performed for 14 meridional sections at $155^{\circ} \mathrm{W}, 150^{\circ} \mathrm{W}, 140^{\circ} \mathrm{W}, 130^{\circ} \mathrm{W}, 100^{\circ} \mathrm{W}$, $76^{\circ} \mathrm{W}, 20^{\circ} \mathrm{W}, 6^{\circ} \mathrm{E}, 10^{\circ} \mathrm{E}, 55^{\circ} \mathrm{E}, 60^{\circ} \mathrm{E}, 90^{\circ} \mathrm{E}, 100^{\circ} \mathrm{E}$, $120^{\circ} \mathrm{E}$ in the area where the ocean depth is at least $3000 \mathrm{~m}$. The areas of the Drake Passage and the southwestern Argentine Basin, the transport through which has become a verification standard of the ACC models, were not included, due to horizontally very coarsely resolved data in this topographically restricted area.

The calculated geostrophic transport $Q_{g}=\int u_{g} d S$ and its longitudinal distribution $q_{g}=\int_{H_{0}}^{H} u_{g} d z$, as well as the respective corrected transports $Q=\int\left(u_{g}+u_{a g}\right) d S$, $q=\int_{H_{0}}^{H}\left(u_{g}+u_{a g}\right) d z$, where $S$ denotes the rectangular area of the selected meridional section between the depths $H_{0}=100 \mathrm{~m}$ and $H=3000 \mathrm{~m}$ in the latitude band from $30^{\circ} \mathrm{S}$ to the southward integration limit, are shown in Fig. 1 and Fig. 2. The selected value of $H$ coincides with the reference depth applied in a variety of the ACC transport estimates (Whitworth et al., 1982; Orsi et al., 1995). The southward integration limits for different longitudinal sections coincide with the southern boundary of the ACC, suggested by Orsi et al. (1995), leaving out the Antarctic surface water together with the areas of the Weddell and Ross gyres. All calculations correspond to $b=10^{3}$, which implies the maximum of $b H\left(\rho / \rho_{0}\right)_{, z}$ to be the of order of 1 .

The calculated geostrophic transport $Q_{g}$ varies around $100 \mathrm{~Sv}$ for all selected meridional sections (Fig. 1). Roughly the same geostrophic transport values have been estimated in several investigations for the Drake Passage (Whitworth et al., 1982) and for the entire Southern Ocean (Orsi et al., 1995), therefore it is assumed that the calculated corrected transports estimated for the same meridional sections are related to the ACC without any substantial contribution from subtropical gyres to the north of the ACC. The corrected transport $Q$, calculated for $A=6 \cdot 10^{-4} \mathrm{~m} \mathrm{~s}^{-1}$ and $A=2 \cdot 10^{-3} \mathrm{~m} \mathrm{~s}^{-1}$, exceeds $Q_{g}$ at all selected longitudes, with the zonal dependence of the corrected transport $Q=Q(\varphi)$ following roughly the pattern of geostrophic transport $Q_{g}=Q_{g}(\varphi)$ (Fig. 1). The meridional distributions of transport $q_{g}=q_{g}(\vartheta)$ and $q=q(\vartheta)$ at longitudes $\varphi=100^{\circ} \mathrm{W}$, 
$20^{\circ} \mathrm{W}$ and $\varphi=55^{\circ} \mathrm{E}$ calculated for $A=6 \cdot 10^{-4} \mathrm{~m} \mathrm{~s}^{-1}$ are shown in Fig. 2a and Fig. 2b. The calculations show an increase in $Q$ with respect to $Q_{g}$, which results from an increase in the current velocity on the southern side of each current branch with bell-shaped velocity distribution and a decrease in the current velocity on the northern side of each branch. The former effect exceeds the latter and, as a result, the total volume transport increases. The increased transport is accompanied by a shift in the corrected velocity maximums to the south if compared with the estimated geostrophic velocity maximums. The indicated southward shifts in the velocity maximums and the increased ACC volume transport are interrelated, therefore the shifts, if detected from observations, would predict the turbulence-generated baroclinic constituent of the ACC volume transport.

\section{Conclusions}

The total ACC volume transport estimated in the Drake Passage and in the southwestern Argentine Basin from the direct velocity measurements exceeds the volume transport predicted by the geostrophic balance in the same region. The difference in the estimates has been traditionally explained by a barotropic eastward flow constituent. The model suggested in the paper, accounting for the effect of the rotational viscosity inherent to the turbulence, with a preferred orientation of eddy rotation, offers another explanation for the observed difference. The model predicts an increase in the ACC volume transport, and includes a specific criterion to distinguish between the turbulence-generated baroclinic flow constituent, on the one hand, and the geostrophic, as well as barotropic transport constituents, on the other hand. The criterion is the southward shift of the velocity maximums of the ACC branches relative to the respective velocity maximums predicted by the geostrophic balance.

Acknowledgements. Topical Editor S. Gulev thanks two referees for their help in evaluating this paper.

\section{References}

Arhan, M., Naveira Garabato, A. C., Heywood, K. J., and Stevens, D. P.: The Antarctic Circumpolar Current between the Falkland islands and South Georgia, J. Phys. Oceanogr., 32, 1914-1931, 2002.

Baker, D. J.: A note on Sverdrup balance in the Southern Ocean, J. Mar. Res., 40, suppl. 21-26, 1982.

Fofonoff, N. P. and Millard Jr., R. C.: Algorithms for computation of fundamental properties of seawater, UNESCO Technical Papers in Marine Science, 44, 53 pp., 1983.

Gille, S. T.: The Southern Ocean momentum balance: evidence for topographic effects from numerical model output and altimeter data, J. Phys. Oceanogr., 27, 2219-2232, 1997.

Gnanadesikan, A. and Hallberg, R. W.: On the relationship of the Circumpolar Current to Southern Hemisphere winds in coarse-
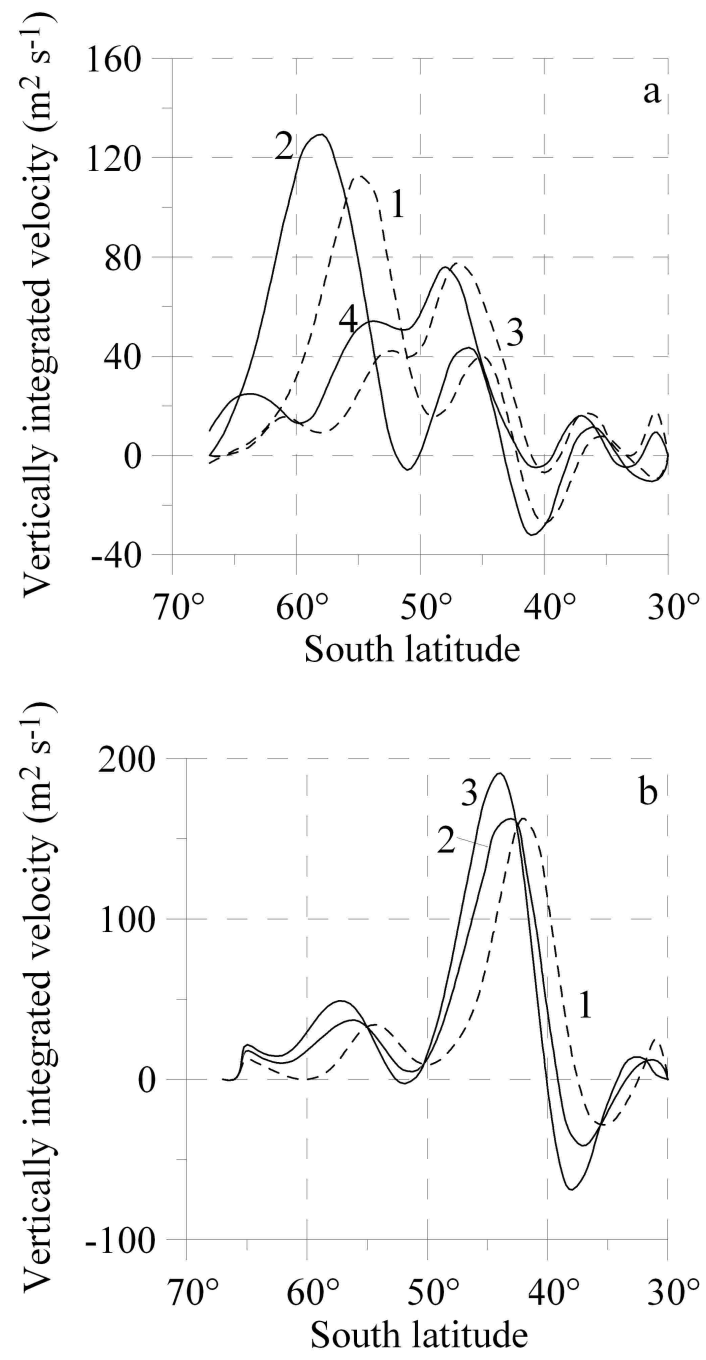

Fig. 2. The meridional distribution of the eastward baroclinic velocity integrated vertically from 100 to $3000 \mathrm{~m}$ (a) along longitudes $150^{\circ} \mathrm{W}$ (curves 1,2 ) and $20^{\circ} \mathrm{W}$ (curves 3,4) for the pure geostrophic estimate $q_{g}$ with $A=0$ (curves 1,3 ) and for the corrected estimate $q$ with $b=10^{3}, A=6 \cdot 10^{-4} \mathrm{~m} \cdot \mathrm{s}^{-1}$ (curves 2, 4); (b) along longitude $55^{\circ} \mathrm{E}$ for pure geostrophic estimate with $A=0$ (curve 1) and for the corrected estimate $q$ with $b=10^{3}, A=6 \cdot 10^{-4}$ $\mathrm{m} \cdot \mathrm{s}^{-1}$ (curve 2) and $b=10^{3}, A=10^{-3} \mathrm{~m} \cdot \mathrm{s}^{-1}$ (curve 3 ).

resolution ocean models, J. Phys. Oceanogr., 30, 2013-2034, 2000.

Heinloo, J.: Formulation of turbulence mechanics, Phys. Review E, 69, 056317, 2004.

Heinloo, J. and Toompuu, A.: Antarctic Circumpolar Current as a density-driven flow, Proc. Estonian Acad. Sci. Phys. Math., 53, 252-265, 2004

Hellerman, S. and Rosenstein, M.: Normal monthly wind stress over the World Ocean with error estimates, J. Phys. Oceanogr., 13, 1093-1104, 1983.

Hofmann, E. E.: The large-scale horizontal structure of the Antarctic Circumpolar Current from FGGE drifters, J. Geophys. Res., 90, 7087-7097, 1985. 
Johnson, G. C. and Bryden, H. L.: On the size of the Antarctic Circumpolar Current, Deep-Sea Res., 36, 39-53, 1989.

Karsten, R., Jones, H., and Marshall, J.: The role of eddy transfer in setting the stratification and transport of a circumpolar current, $\mathrm{J}$. Phys. Oceanogr., 32, 39-54, 2002.

Kolmogoroff, A. N.: The local structure of turbulence in incompressible viscous fluids for very large Reynolds numbers, C. R. Acad. Sci. URSS, 30, 376-387, 1941.

Mestaz-Nunez, A. M., Chelton, D. B., and DeSzoeke, R. A.: Evidence of time-dependent Sverdrup circulation in the South Pacific from the Seasat scatterometer and altimeter, J. Phys. Oceanogr., 22, 934-943, 1992.

Morrow, R., Church, J., Coleman, R., Chelton, D., and White, N.: Eddy momentum flux and its contribution to the Southern Ocean momentum balance, Nature, 357, 482-484, 1992.

Munk, W. H. and Palmén, E.: Note on the dynamics of the Antarctic Circumpolar Current, Tellus, 3, 53-56, 1951.

Niiler, P.: The World Ocean surface circulation, in: Ocean Circulation and Climate: Observing and Modelling the Global Ocean, edited by: Siedler, G., Church, J., and Gould, J., Academic Press, 193-204, 2001.

Nowlin Jr., W. D. and Klinck, J. M.: The physics of the Antarctic Circumpolar Current, Rev. Geophys., 24, 469-491, 1986.

Olbers, D.: Comments on "On the obscurantist physics of 'form drag' in theorizing about the Circumpolar Current", J. Phys. Oceanogr., 28, 1647-1654, 1998.

Olbers, D., Gouretski, V., Seiss, G., and Schroeter, J.: Hydrographic Atlas of the Southern Ocean, Alfred-Wegener-Institut für Polarund Meeresforschung, Bremerhaven, 1992.
Orsi, A. H., Whitworth III, T., and Nowlin Jr., W. D.: On the meridional extent and fronts of the Antarctic Circumpolar Current, Deep-Sea Res. I, 42, 641-673, 1995.

Phillips, H. E. and Rintoul, S. R.: Eddy variability and energetics from direct current measurements in the Antarctic Circumpolar Current south of Australia, J. Phys. Oceanogr., 30, 3050-3076, 2000.

Pedlosky, J.: Geophysical fluid dynamics, Springer-Verlag, 624 pp., 1982.

Richardson, L. F. : Weather prediction by numerical process, Cambridge Univ. Press, Cambridge, 1922.

Rintoul, S. R., Hughes, C. W., and Olbers, D.: The Antarctic Circumpolar Current System, in: Ocean Circulation and Climate: Observing and Modeling the Global Ocean, edited by: Siedler, G., Church, J., and Gould, J., Academic Press, 271-301, 2001.

Tansley, C. E. and Marshall, D. P.: On the dynamics of wind-driven circumpolar currents, J. Phys. Oceanogr., 31, 3258-3273, 2001.

Warren, B., LaCasce, J., and Robbins, P. A.: On the obscurantist physics of "form drag" in theorizing about the Circumpolar Current, J. Phys. Oceanogr., 26, 2297-2301, 1996.

Whitworth III, T., Nowlin Jr., W. D., and Worley, S. J.: The net transport of the Antarctic Circumpolar Current trough Drake Passage, J. Phys. Oceanogr., 12, 960-971, 1982.

Wyrtki, K.: The Antarctic Circumpolar Current and the Antarctic Polar Front, Deutsche Hydrographische Zeitschrift, 13, 153-174, 1960. 